HNO 2016 $64: 352-353$

DOI 10.1007/s00106-016-0171-5

Online publiziert: 24. Mai 2016

๑) Springer-Verlag Berlin Heidelberg 2016

CrossMark

\section{H.-G. Struck • J. Heichel}

Universitätsklinik und Poliklinik für Augenheilkunde, Martin-Luther-Universität Halle-Wittenberg,

Universitätsklinikum Halle (Saale), Halle (Saale), Deutschland

\title{
Ableitende Tränenwege im Fokus von Ophthalmo- und Rhinochirurgen
}

Die ableitenden Tränenwege durchziehen von den Puncta lacrimalia im inneren Lidwinkel bis zu ihrer Einmündung in den unteren Nasengang ein fächerübergreifendes Grenzgebiet. Kardinalsymptom ihrer Verlegung ist die Epiphora, bei intra- und postsakkalen Stenosen kombiniert mit einer akuten oder chronischen Dakryozystitis und deren Komplikationen.

Während die Basisdiagnostik bei Tränenwegserkrankungen Sache des Augenarztes ist, sind an der interdisziplinären Spezialdiagnostik stets die HNO-Ärzte und bei Bedarfauch die Radiologen beteiligt. Traditionell ergänzen sich Ophthalmo- und Rhinochirurgen in der operativen Sanierung von Verschlüssen der ableitenden Tränenwege und ursächlicher pathologischer Veränderungen im Nasen- und Nasennebenhöhlenbereich.

\section{》) Ophthalmo- und Rhino- chirurgen ergänzen sich bei der Tränenwegssanierung}

Ziel dieses Themenhefts ist es, die ganze Bandbreite der aktuellen Möglichkeiten in Diagnostik und Therapie der häufigsten Krankheitskomplexe vergleichend darzustellen und zukunftsweisende Entwicklungen aufzuzeigen. Die Grundlage für diese klinischen Betrachtungen liefert der Beitrag von F. Paulsen et al. zur „Anatomie und Physiologie der ableitenden Tränenwege“. Durch ihn wird auch das Verständnis für die Entstehung der konnatalen Dakryostenose gefördert, deren Ursachen und Diagnostik aus ophthalmologischer Sicht - verbunden mit einem aktuell erprobten therapeutischen Stufenkonzept - von J. Heichel et al. in einer prägnanten Übersichtsarbeit dargestellt werden. Ergänzend hierzu werden von dem gleichen Autor die speziellen Rahmenbedingungen bei der Betreuung der behandelten Kinder und deren Angehörigen in einer Originalarbeit $\mathrm{zu}$ medizinpsychologischen Aspekten bei der Behandlung kindlicher Tränenabflussstörungen mit der Darstellung von Einflussfaktoren auf die elterliche Bewertung des eigenen und kindlichen Stresserlebens beschrieben sowie in einem Beitrag der Rubrik „Bild und Fall“" auf das Auftreten von angeborenen Tränenwegstenosen im Rahmen von Systemerkrankungen bzw. Syndromen hingewiesen.

Den erworbenen Stenosen der ableitenden Tränenwege liegt oft ein multifaktorielles Geschehen zugrunde. Auf die besondere Rolle von Tumoren, die potenziell maligne sein können und bei langer Anamnese und atypischen klinischen Zeichen oft erst spät diagnostiziert und behandelt werden, gehen C. von Goszinski et al. in ihrer Übersichtarbeit umfassend ein. Eine weitere Übersichtsarbeit ist der operativen Therapie der erworbenen Tränenwegsverschlüsse gewidmet.

So vergleichen C. Ginzkey und R. Mlynski den aktuellen Entwicklungsstand der endonasalen Dakryozystorhinostomie mit dem anderer Operationstechniken und liefern überzeugende Belege für die Vervollkommnung dieser Operationstechnik und die damit zu erzielenden funktionellen Ergebnisse.

In der Arbeit „Konkremente und iatrogene Fremdkörper der ableitenden Tränenwege - therapeutische Empfehlungen“" setzen sich J. Heichel et al. mit dieser Problematik auseinander. In dieser retrospektiven Studie waren von 1451 konsekutiven operativen Eingriffen an den ableitenden Tränenwegen 232 (etwa $16 \%$ ) von dieser speziellen pathologischen Veränderung betroffen und wurden zu deren Auswirkung auf Krankheitsverlauf und Operationstechnik bewertet.

\section{》) In Halle gibt es eine ständige interdisziplinäre Tränenwegskonferenz}

Die abschließende Originalarbeit von $H$.G. Struck et al. ist der 2013 am Universitätsklinikum Halle etablierten ständigen interdisziplinären Tränenwegskonferenz gewidmet, in der die langjährigen Erfahrungen von Ophthalmo- und Rhinochirurgen planmäßig zusammengeführt werden. Diese unseres Wissens bisher neuartige Form der Zusammenarbeit beider Fachgebiete dient auch der Fort- und Weiterbildung. Eine erste Analyse der Ergebnisse ihrer Tätigkeit bei einem $\mathrm{Pa}$ tientenkollektiv mit Erkrankungen der ableitenden Tränenwege und erhöhtem Gefährdungsgrad wird von den Autoren 
beider Fachgebiete in diesem Themenheft gemeinsam mit den weiteren Beiträgen einem breiten Kollegenkreis vorgestellt.

Halle, 23. März 2016

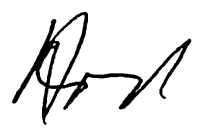

Hans-Gert Struck

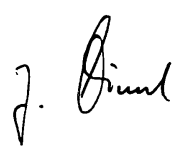

Jens Heichel

\section{Korrespondenzadresse}

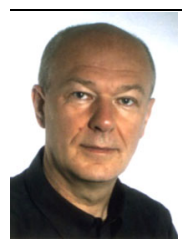

Prof. Dr. H.-G. Struck

Universitätsklinik und

Poliklinik für Augenheilkunde,

Martin-Luther-Universität

Halle-Wittenberg,

Universitätsklinikum Halle

(Saale)

Ernst-Grube-Straße 40,

06120 Halle (Saale),

Deutschland

hans-gert.struck@uk-halle.de

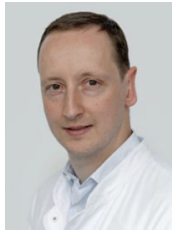

\section{Dr. J. Heichel}

Universitätsklinik und Poliklinik für Augenheilkunde, Martin-Luther-Universität Halle-Wittenberg, Universitätsklinikum Halle (Saale) Ernst-Grube-Straße 40, 06120 Halle (Saale), Deutschland

Interessenkonflikt. H.-G. Struckund J. Heichel geben an, dass kein Interessenkonflikt besteht.

\section{Madaitorial}

Unsere ausführlichen Autorenleitfäden und Musterbeiträge finden Sie online unter „Hinweise für Autoren" auf/Author guidelines are available at: www.HNO.springer.de

\section{Manuskripteinreichung}

(alle Rubriken außer CME Zertifizierte Fortbildung):

Bitte reichen Sie Ihre Manuskripte ausschließlich über das Online-System „Editorial Manager" ein. Wählen Sie hierzu auf der Zeitschriftenhomepage www.HNO.springer.de den Navigationspunkt „Für Autoren“ und anschließend „Manuskript online einreichen".

Bitte beachten Sie, dass bei jeder Einreichung das Formular „Angaben zum Interessenkonflikt" beigelegt sein muss.

Nach der erstmaligen Registrierung finden Sie unter „Author Login“ alle notwendigen Hinweise, wie Sie Ihren Beitrag hochladen können.

Bei Fragen zur Einreichung wenden Sie sich bitte an:

Dagmar Lorenz

Springer-Verlag GmbH, Tiergartenstr. 17, 69121 Heidelberg,

Tel.: +49 (0)6221/487-8167, Fax: -68167

E-Mail: dagmar.lorenz@springer.com

Bitte beachten Sie, dass Artikel der Rubrik CME Zertifizierte Fortbildung weiterhin per E-Mail an die zuständigen Herausgeber eingereicht werden. Die Adressen finden Sie unter www.HNO.springer.de 